\title{
Association of FOXJ1 polymorphisms with systemic lupus erythematosus and rheumatoid arthritis in Korean population
}

\author{
Chun-Shi Li ${ }^{1,3}$, Qinggao Zhang ${ }^{4}$, Mi-Kyoung Lim ${ }^{5}$, \\ Dong-Hyuk Sheen ${ }^{5}$, Seung-Cheol Shim ${ }^{5}$, \\ Ji-Young Kim ${ }^{5}$, Shin-Seok Lee ${ }^{6}$, Ki-Jung Yun ${ }^{2}$, \\ Hyung-Bae Moon ${ }^{2}$, Hun-Taeg Chung ${ }^{1,7}$ \\ and Soo-Cheon Chae ${ }^{2,7}$ \\ ${ }^{1}$ Genome Research Center for Immune Disorders \\ ${ }^{2}$ Department of Pathology \\ School of Medicine, Wonkwang University \\ Iksan 570-749, Korea \\ ${ }^{3}$ Department of Pharmacology \\ ${ }^{4}$ Department of Microbiology and Immunology \\ Yanbian University Medical College \\ 133000 Yianji, Jilin, China \\ ${ }^{5}$ Division of Rheumatology \\ Department of Internal Medicine \\ Eulji University School of Medicine \\ Daejeon 301-831, Korea \\ ${ }^{6}$ Division of Rheumatology \\ Department of Internal Medicine \\ Chonnam National University Medical School \\ Gwangju 501-746, Korea \\ ${ }^{7}$ Corresponding authors: Tel, 82-63-850-6793; \\ Fax, 82-63-852-2110; E-mail, chaesc@wonkwang.ac.kr (for S.C.C.) and \\ Tel, 82-63-850-6762; Fax, 82-63-851-5066; \\ E-mail, htchung @ wonkwang.ac.kr (for H.T.C.)
}

\section{Accepted 7 November 2007}

Abbreviations: FOXJ1, forkhead-box J1; RA, rheumatoid arthritis; $\mathrm{SBE}$, single-base extension; SLE, systemic lupus erythematosus; SNP, single nucleotide polymorphism

\footnotetext{
Abstract

The forkhead-box J1 (FOXJ1) transcription factor could suppress a spontaneous activation of $T$ cells and $B$ cells through an induction of $I_{\kappa} B \beta$ that results in repression of NF-KB activity. In Foxj1 deficiency mice, systemic autoimmune inflammation is quite common symptom. Therefore, deregulated Foxj 1 is supposed to be associated with autoimmune diseases and/or other inflammatory diseases. Previously, we identified that polymorphisms of human FOXJ1 gene (g. $-460 \mathrm{C}>T$, g.1805G $>T$ and $g .3375 G>C$ ) are associated with allergic rhinitis in a Korean population. In present study, we compared the genotype and allele frequencies of
}

these SNPs between healthy controls and systemic lupus erythematosus (SLE) or rheumatoid arthritis (RA) patients. We also investigated the relationships between each genotype and the expression levels of antinuclear antibodies in SLE patients, and rheumatoid factor and anti-cyclic citrullinated peptide in RA patients. The frequencies of haplotypes constructed by these FOXJ1 SNPs were compared between controls and SLE (or RA) patients. The results of genotype and allele analysis showed that the prevalence of polymorphism g.3375G $>C$ was associated with the susceptibility of SLE ( $P=\mathbf{0 . 0 0 7 2}$ and 0.0042 , respectively). But no significant association was found with RA. In the haplotype analysis, however, the main CGG showed a weak association between controls and RA patients $(P=0.048)$.

Keywords: autoantibodies; FOXJ1 protein, human; polymorphism, genetic; lupus erythematosus, systemic; rheumatoid arthritis

\section{Introduction}

Systemic lupus erythematosus (SLE) and rheumatoid arthritis (RA) are the representative autoimmune diseases. These diseases are arisen by the complex interaction between multiple genetic factors and environmental factors (Kotzin, 1996; Gregersen, 1999). The clinical manifestations of RA are thought to be triggered by over-activation of helper T1 (Th1) cell (Simon et al., 1994; Dolhain et al., 1996) while SLE is characterized by elevation of both Th1 and Th2 cells. The predominance of Th1 and/or Th2 cells depends on the stage of disease development (Akahoshi et al., 1999; Chang et al., 2002). RA and SLE are most commonly presented in women. Particularly, the frequency of SLE between female and male is 9 to1 (Hochberg, 1997). Rheumatoid factor (RF) has been widely used as a screening test for patients with arthritis. RF is prognostically useful to correlates with functional (Van der Heide et al., 1995) and outcomes in both RA and early inflammatory polyarthritis (Harrison et al., 1999). More recently a highly specific autoantibody system has been described for RA, in which the synthetic cyclic citrullinated peptide (CCP) with deiminated arginines is used as the antigen for anti-CCP antibodies (Schellekens et al., 2000). 
Foxj1 (also known as HFH-4 and FKHL-13) is a member of the forkhead-box (FOX) gene family, which encodes transcription factors composed of with a conserved DNA binding motif known as the forkhead domain (Weigel and Jackle, 1990). Recent studies suggest more than 100 forkhead transcription factors classified into 17 subgroups, from FOXA to FOXQ, of FOX family (Kaestner et al., 2000). Fox family takes part in diverse biological functions including development, metabolism, aging, and cancer (Carlsson and Mahlapuu, 2002; Tran et al., 2003; Coffer and Burgering, 2004). Foxj1 is expressed mainly in tissues of ciliated respiratory cells, reproductive and central nervous systems. Furthermore, it is regarded as having association with ciliated cell development. In most cases, the mutation of foxj1 gene resulted in an absence of cilia in the tissues of mice models (Hackett et al., 1995; Lim et al., 1997; Chen et al., 1998; Blatt et al., 1999). Foxj1 also plays critical roles in immune system (Lin et al., 2004, 2005). Foxj1 is a repressor of NF- $\kappa \mathrm{B}$, a transcription factor responsible for a great variety of pro-inflammatory cytokines production. Foxj1-deficient mice showed multi-organ inflammation. And the expression of foxj1 was decreased in lupus prone mice (Lin et al., 2004). On the basis of the rationale, foxj1 has been postulated as a potential candidate gene that is associated with autoimmune and (or) inflammatory diseases.

As we previously identified, the seven SNPs, [g. $-460 C>T$ (rs880213) and g. $-342 G>C$ (rs880212 ) in promoter region, g.1164G $>C, g .1805 G$ $>T$ (rs1868823), g.1824C $>G$ and $g .1849 G>C$ (rs1868824) in intron1, and $g .3375 G>C$ (rs3192453) in 3'-UTR region, in human FOXJ1 gene and reported that the $g .-460 C>T, g .1805 G>T$ and g.3375G $>C$ polymorphisms in FOXJ1 gene were associated with susceptibility to allergic rhinitis ( $\mathrm{Li}$ et al., 2006). To determine whether these polymorphisms are associated with the susceptibility of autoimmune diseases such as SLE and RA, we compared the genotype and allele frequencies between healthy controls and patients having RA or SLE. We also investigated the relationships of each genotype with some clinical characteristics, such as RF and CCP antibody for RA, and anti-nuclear antibodies (ANA) for SLE. We further analyzed the differences of haplotype distributions by these SNPS between controls and patients with RA or SLE.

\section{Materials and Methods}

\section{Patients and genomic DNA}

Blood samples were obtained from 418 controls
(260 males and 158 females), 204 SLE patients (13 males and 191 females) and 376 RA patients (70 males and 306 females). The mean age of the control, SLE and RA groups were 38.3 years, 34.8 years and 53.8 years, respectively. Healthy controls were recruited from individuals who took comprehensive examination in Wonkwang University Hospital. The RA and SLE patients were recruited from outpatient clinic at Eulji University Hospital and Chonnam National University Hospital. RA and SLE were diagnosed according to the criteria of the American Rheumatism Association, and the American College of Rheumatology (ACR), respectively (Arnett et al., 1988; Hochberg, 1997). CCP and RF levels in RA patients and ANA levels in SLE were determined in a routine laboratory at the Eulji University Hospital and Chonnam National University Hospital. Genomic DNA was extracted from leukocytes in peripheral blood by a standard phenol-chloroform method or by Genomic DNA Extraction kit (iNtRON Biotechnology, Korea) according to manufacturer's directions.

\section{PCR}

The previously used three primer pairs were used for amplification of the FOXJ1 genes containing g. $-460 C>T$, g.1805G $>T$ and $g .3375 G>C$ polymorphisms (Li et al., 2006). PCR was performed in a $20 \mu \mathrm{l}$ reaction volume and reaction mixture were prepared by previously described condition (Chae et al., 2004). Amplification was carried out in a GeneAmp PCR system 9700 thermocycler (Applied Biosystem) at $95^{\circ} \mathrm{C}$ for $5 \mathrm{~min}$ to pre-denature the template DNA, followed by 30 cycles of denaturation at $98^{\circ} \mathrm{C}$ for $10 \mathrm{~s}$, annealing at $68^{\circ} \mathrm{C}$ for $30 \mathrm{~s}$ and extension at $72^{\circ} \mathrm{C}$ for 2.0 or $2.5 \mathrm{~min}$, finally, extension for another $10 \mathrm{~min}$ at $72^{\circ} \mathrm{C}$ to end the reaction. The PCR products were purified by PCR Purification Kit (Millipore) and were used as template DNA for direct sequencing and single base extension.

\section{Genotyping}

Genotyping was performed by single-base extension (SBE) using the $A B I$ Prism ${ }^{\circledR}$ SNaPshot $^{\mathrm{TM}}$ Multiplex kit (Applied Biosystems, Foster city, CA). The previously used three SBE primers were used in this study for genotyping of FOXJ1 SNPs in RA patients, SLE patients and controls (Li et al., 2006). The SBE reaction mixture was prepared according to the manufacturer's instructions. The primer extension reaction was performed at $96^{\circ} \mathrm{C}$ for 1 min, followed by 25 cycles at $96^{\circ} \mathrm{C}$ for $5 \mathrm{~s}, 55^{\circ} \mathrm{C}$ for $30 \mathrm{~s}$, and $60^{\circ} \mathrm{C}$ for $30 \mathrm{~s}$. To clean up the primer 
extension reaction, $1.5 \mathrm{U}$ of calf intestinal alkaline phosphatase (CIP, New England BioLabs) was added to the reaction mixture, and the mixture was incubated at $37^{\circ} \mathrm{C}$ for $60 \mathrm{~min}$, followed by $15 \mathrm{~min}$ at $72^{\circ} \mathrm{C}$, for purposes of enzyme inactivation. The purified extension products were added to $\mathrm{Hi}-\mathrm{Di}$ formamide (Applied Biosystems) according to the recommendations of the manufacturer. The mixture was incubated at $95^{\circ} \mathrm{C}$ for $5 \mathrm{~min}$, followed by 5 min on ice, and then electrophoresis was performed, using the ABI Prism 3100 Genetic Analyzer. The results were analyzed using the ABI Prism GeneScan and Genotyper software (Applied Biosystems).

\section{Statistic analysis}

$\chi^{2}$ tests were used to estimate the Hardy- Weinberg equilibrium (HWE). Linkage Disequilibrium (LD) analyses by pair-wise comparison of biallelic loci and the haplotype frequencies of FOXJ1 for multiple loci were estimated using the expectation maximization (EM) algorithm with SNPAlyze software (DYNACOM, Japan). Logistic regression analyses were used to calculate odds ratios $(95 \%$ confidence interval), and ANOVA was applied in order to analyze differences among the each genotypes, and RF, CCP levels for RA patients or ANA and anti-RNP levels for SLE patients. Cutoff value of $R F$ is $18 \mathrm{IU} / \mathrm{ml}$ and that of anti-CCP antibody is $5 \mathrm{U} / \mathrm{ml}$. A $P$-value of less than 0.05 was considered to indicate statistical significance.

\section{Results}

We previously identified seven SNPs in human FOXJ1 gene and the three polymorphisms (g. $-460 C>T, g .1805 G>T$ and $g .3375 G>C$ ) were associated with susceptibility to allergic rhinitis ( $\mathrm{Li}$ et al., 2006). In our present study, we attempted to find out whether these polymorphisms were further associated with genetic predisposition of SLE and RA. The genotypes of these polymorphisms were determined in 204 unrelated SLE patients, in 376 unrelated RA patients and in 418 unrelated controls without SLE and RA. All genotype frequencies were in Hardy-Weinberg equilibrium (HWE) by $\chi^{2}$ tests (data not shown). In comparison between SLE (or RA) patients and the controls, the genotype and allele frequencies of each polymorphism were analyzed (Table 1). While the genotype and allele frequencies of these SNPs, in RA patients, were no significant association. The genotype and allele frequencies of $\mathrm{g} .3375 \mathrm{G}>\mathrm{C}$ polymorphism, in SLE patients, were significantly different from those of controls group $(P=0.007$ and 0.004 , respectively). Among female, we further analyzed the $P$ values for $g .-460 C>T, g .1805 G>T$ and g.3375G $>C$ polymorphisms between the controls and RA (or SLE) patients, because both RA and SLE patients are predominantly female in compare with control subject (Table 2). As we expected, however, our results indicate that gender is a minor factor in the association of RA patients. On the whole, in SLE female patients, the genotype and

Table 1. Genotype and allele analysis of the polymorphisms in FOXJ1 gene between patients with SLE or RA and controls.

\begin{tabular}{|c|c|c|c|c|c|c|}
\hline \multirow{2}{*}{ Position ${ }^{a}$} & \multirow{2}{*}{ Genotype } & \multirow{2}{*}{$\begin{array}{c}\text { Control } \\
n(\%)\end{array}$} & \multirow{2}{*}{$\begin{array}{c}\mathrm{RA} \\
n(\%)\end{array}$} & \multirow{2}{*}{$\begin{array}{l}\text { SLE } \\
n(\%)\end{array}$} & \multicolumn{2}{|c|}{$P^{b}$} \\
\hline & & & & & vs. RA & SLE \\
\hline g. $-460 C>T$ & $\mathrm{CC}$ & $321(76.8)$ & $288(77.0)$ & $137(71.7)$ & 0.996 & 0.284 \\
\hline \multirow[t]{4}{*}{ (rs880213) } & $\mathrm{CT}$ & $90(21.5)$ & $80(21.4)$ & $48(25.1)$ & & \\
\hline & TT & $7(1.7)$ & $6(1.6)$ & $6(3.1)$ & & \\
\hline & $\mathrm{C}$ & 732 (87.6) & $656(87.7)$ & 322 (84.3) & 0.939 & 0.125 \\
\hline & $\mathrm{T}$ & $104(12.4)$ & $92(12.3)$ & 60 (15.7) & & \\
\hline g. $1805 \mathrm{G}>\mathrm{T}$ & GG & $294(70.5)$ & 269 (71.7) & $132(65.3)$ & 0.881 & 0.283 \\
\hline \multirow[t]{4}{*}{ (rs1868823) } & GT & $114(27.3)$ & 97 (25.9) & $62(30.7)$ & & \\
\hline & TT & $9(2.2)$ & $9(2.4)$ & $8(4.0)$ & & \\
\hline & $G$ & 702 (84.2) & $635(84.7)$ & $328(80.7)$ & 0.835 & 0.146 \\
\hline & $\mathrm{T}$ & $132(15.8)$ & 115 (15.3) & 78 (19.3) & & \\
\hline g. $3375 G>C$ & GG & $318(77.0)$ & $289(77.7)$ & $135(67.2)$ & 0.760 & 0.007 \\
\hline \multirow[t]{4}{*}{ (rs3192453) } & $\mathrm{GC}$ & $88(21.3)$ & 79 (21.2) & 65 (32.3) & & \\
\hline & $\mathrm{CC}$ & $7(1.7)$ & $4(1.1)$ & $1(0.5)$ & & \\
\hline & G & 724 (87.7) & $657(88.3)$ & 335 (83.3) & 0.699 & 0.004 \\
\hline & C & $102(12.3)$ & $87(11.7)$ & 67 (16.7) & & \\
\hline
\end{tabular}

${ }^{\mathrm{a}}$ Calculated from the translation start site.

balue was determined by Fisher's exact test or $\chi^{2}$ test from $2 \times 2$ contingency table. 
allele frequencies of $\mathrm{g} .3375 \mathrm{G}>\mathrm{C}$ polymorphism were not significantly different from that of female control groups $(P=0.273$ and 0.185 , respectively). On the contrary, in SLE male patients, the polymorphisms of SLE (g.1805G $>T$ and $g .3375 G>C$ ) are significantly different from the male control group $(P=0.023$ and 0.003 respectively, data not shown). Therefore, we partially suggest that the association of the SNPs of FOXJI ( $g .1805 \mathrm{G}>T$ and g.3375G $>C$ ) could be affected by the gender of patients. On the other hand, to define a possible correlation between polymorphisms and clinical features of SLE and RA, we further analyzed each genotype of these SNPs with ANA in SLE patients and anti-CCP and RF levels in RA patients (Table 3). We found that these SNPS in RA and SLE patients have no significant association with the levels of RF and anti-CCP, and ANA, respectively (Table 3). We analyzed each genotype of these SNPs according to positivity of RF and anti-CCP in RA patients RA, and found that there are no significant associations (data not shown). We also evaluated the haplotype frequencies among g. $-460 C>T$, g.1805G $>T$ and g.3375G $>C$ polymorphisms in both healthy controls and RA patients or SLE patients (Table 4). While three

Table 2. Genotype and allele analysis of the polymorphisms in FOXJ1 gene between the female of RA or SLE patients and controls.

\begin{tabular}{|c|c|c|c|c|c|c|}
\hline \multirow{2}{*}{ Position $^{a}$} & \multirow{2}{*}{ Genotype } & \multirow{2}{*}{$\begin{array}{c}\text { Control } \\
n(\%)\end{array}$} & \multirow{2}{*}{$\begin{array}{c}\mathrm{RA} \\
n(\%)\end{array}$} & \multirow{2}{*}{$\begin{array}{l}\text { SLE } \\
n(\%)\end{array}$} & \multicolumn{2}{|c|}{$P^{b}$} \\
\hline & & & & & vs. RA & SLE \\
\hline \multirow[t]{5}{*}{ g. $-460 \mathrm{C}>\mathrm{T}$} & $\mathrm{CC}$ & $116(74.4)$ & $227(76.9)$ & $131(72.8)$ & 0.736 & 0.629 \\
\hline & CT & $38(24.4)$ & $63(21.4)$ & $44(24.4)$ & & \\
\hline & TT & $2(1.2)$ & $5(1.7)$ & $5(2.8)$ & & \\
\hline & $C$ & 270 (86.5) & $517(87.6)$ & $306(85.0)$ & 0.675 & 0.583 \\
\hline & $\mathrm{T}$ & $42(13.5)$ & 73 (12.4) & $54(15.0)$ & & \\
\hline \multirow[t]{5}{*}{ g.1805G $>\mathrm{T}$} & GG & $110(70.5)$ & $213(72.2)$ & $126(66.7)$ & 0.623 & 0.446 \\
\hline & GT & $44(28.2)$ & 75 (25.4) & 57 (30.2) & & \\
\hline & $\mathrm{TT}$ & $2(1.3)$ & $7(2.4)$ & $6(3.1)$ & & \\
\hline & $\mathrm{G}$ & 264 (84.6) & 501 (84.9) & $309(81.7)$ & 0.922 & 0.359 \\
\hline & $\mathrm{T}$ & $48(15.4)$ & $89(15.1)$ & 69 (18.3) & & \\
\hline \multirow[t]{5}{*}{ g. $3375 G>C$} & GG & 119 (76.8) & $228(77.8)$ & $130(69.1)$ & 0.729 & 0.273 \\
\hline & $\mathrm{GC}$ & 35 (22.6) & $61(20.8)$ & $57(30.3)$ & & \\
\hline & $\mathrm{CC}$ & $1(0.6)$ & $4(1.4)$ & $1(0.5)$ & & \\
\hline & $\mathrm{G}$ & 273 (88.1) & $517(88.2)$ & 317 (84.3) & 1.00 & 0.185 \\
\hline & $C$ & 37 (11.9) & $69(11.8)$ & $59(15.7)$ & & \\
\hline
\end{tabular}

${ }^{a}$ Calculated from the translation start site.

balue was determined by Fisher's exact test or $\chi^{2}$ test from $2 \times 2$ contingency table.

Table 3. Analysis of RF and anti-CCP levels in RA patients, and ANA levels in SLE patients among the each genotype of FOXJ1 SNPs.

\begin{tabular}{|c|c|c|c|c|c|c|c|c|c|c|c|c|c|}
\hline \multirow{2}{*}{ Position $^{a}$} & \multirow{2}{*}{ Genotype } & \multicolumn{3}{|c|}{ RF (1 U/ml) } & \multirow{2}{*}{$P^{b}$} & \multicolumn{3}{|c|}{ Anti-CCP } & \multirow{2}{*}{$P^{b}$} & \multicolumn{3}{|c|}{ ANA } & \multirow{2}{*}{$P^{\mathrm{b}}$} \\
\hline & & $n$ & Mean & SD & & $n$ & Mean & SD & & $n$ & Mean & SD & \\
\hline \multirow[t]{3}{*}{ g. $-460 \mathrm{C}>\mathrm{T}$} & $\mathrm{CC}$ & 270 & 70.0 & 71.0 & 0.37 & 142 & 57.4 & 57.2 & 0.33 & 90 & 2.40 & 0.41 & 0.36 \\
\hline & CT & 78 & 57.3 & 66.1 & & 32 & 58.3 & 53.1 & & 35 & 2.31 & 0.36 & \\
\hline & $\mathrm{TT}$ & 6 & 69.5 & 90.2 & & 4 & 99.9 & 33.4 & & 4 & 2.20 & 0.35 & \\
\hline \multirow[t]{3}{*}{ g.1805G $>\mathrm{T}$} & GG & 253 & 69.6 & 70.8 & 0.18 & 131 & 59.0 & 57.3 & 0.26 & 87 & 2.38 & 0.40 & 0.90 \\
\hline & GT & 93 & 58.8 & 65.3 & & 41 & 52.1 & 52.8 & & 46 & 2.38 & 0.40 & \\
\hline & $\mathrm{TT}$ & 9 & 98.9 & 105.0 & & 6 & 92.0 & 46.3 & & 6 & 2.30 & 0.37 & \\
\hline \multirow[t]{3}{*}{ g. $3375 G>C$} & GG & 271 & 70.9 & 71.8 & 0.12 & 143 & 57.4 & 57.0 & 0.87 & 91 & 2.38 & 0.38 & 0.92 \\
\hline & GC & 77 & 56.4 & 66.0 & & 32 & 59.1 & 54.0 & & 47 & 2.38 & 0.43 & \\
\hline & $\mathrm{CC}$ & 4 & 40.5 & 31.7 & & 2 & - & - & & 0 & - & - & \\
\hline
\end{tabular}

${ }^{a}$ Calculated from the translation start site.

${ }^{b}$ Values were analyzed by ANOVA. 
Table 4. The haplotype frequencies between RA or SLE patients and controls in FOXJ1 SNPS.

\begin{tabular}{|c|c|c|c|c|c|c|c|}
\hline \multicolumn{3}{|c|}{ Haplotype } & \multicolumn{3}{|c|}{ Frequency $^{a}$} & \multicolumn{2}{|c|}{$P^{b}$} \\
\hline g. $-460 \mathrm{C}>\mathrm{T}$ & g.1805G $>\mathrm{T}$ & g. $3375 \mathrm{C}>\mathrm{T}$ & Control & RA & SLE & vs. RA & SLE \\
\hline C & G & G & 0.802 & 0.840 & 0.804 & 0.048 & 0.945 \\
\hline T & T & C & 0.088 & 0.113 & 0.118 & 0.103 & 0.106 \\
\hline C & $\mathrm{T}$ & G & 0.050 & 0.034 & 0.014 & 0.103 & 0.010 \\
\hline $\mathrm{T}$ & $\mathrm{G}$ & $\mathrm{G}$ & 0.014 & 0.004 & 0.000 & 0.033 & 0.025 \\
\hline $\mathrm{T}$ & $\mathrm{G}$ & $\mathrm{C}$ & 0.014 & 0.001 & 0.000 & 0.017 & 0.042 \\
\hline C & $\mathrm{T}$ & C & 0.012 & 0.005 & 0.002 & 0.004 & 0.130 \\
\hline C & $\mathrm{G}$ & $\mathrm{C}$ & 0.011 & 0.003 & 0.027 & 0.086 & 0.088 \\
\hline T & $\mathrm{T}$ & G & 0.009 & 0.000 & 0.035 & 0.404 & 0.001 \\
\hline
\end{tabular}

${ }^{2}$ Values were constructed by EM algorithm with genotyped SNPs.

balues were analyzed by permutation test.

major haplotypes were identified explaining more than $94.0 \%$ of distribution in controls, two major types were identified in SLE (92.1\%) and RA $(95.3 \%)$ patients out of eight possible haplotypes. And the main haplotype, CGG, represented a weak association with RA $(P=0.048)$, the haplotype CTG also showed a significant difference between SLE patients and controls $(P=0.01)$.

\section{Discussion}

The activated Th cells during the developing stage are differentiated into phenotypically and functionally two distinct types of cells, Th1 and Th2 (Mosmann and Coffman, 1989; Abbas et al., 1996). Th1 cells produce cytokines such as IFN- $\gamma$, IL-12 and cytotoxic factor lymphotoxin. They are commonly associated with cell-mediated immune responses against intracellular pathogens and induction of organ-specific autoimmune diseases such as RA (Kuchroo et al., 1995; Abbas et al., 1996). On the other hand, the Th2 cell related cytokines IL-4, IL-5 and IL-10, are known to be associated with atopic and allergic diseases. The balance between Th1 and Th2 cells is critical in the immune response to pathogens, tumor antigens and allergens. Th1 and Th2 cells cross-regulate the differentiation of each other. The predominant induction of Th2 cells inhibits autoimmune diseases such as RA and the predominant induction of Th1 cells inhibits induction of asthma and allergic diseases (Lafaille et al., 1997; Ho and Glimcher, 2002).

Foxj1 is a presumed transcription factor containing DNA binding domain. Foxj1 induces the production of $I_{\kappa} B \beta$ and subsequently suppresses the activity of $N F-\kappa B$, the potent proinflammatory cytokine inducer. Down-regulated expression of
Foxj1 was observed in a lupus prone mice model. Further more, systemic autoimmune inflammation was demonstrated in Foxj1 (-I-) mice (Lin et al., 2004). Under the neutralizing condition, the CD4 ${ }^{+} \mathrm{T}$ cells from Foxj1 (-l-) mice produced more Th1 cytokines than that from Foxj1 (+/+) mice. The phenomena, probably, designates a Th1 bias state (Lin et al., 2004). It suggests that Foxj1 is a possible gene of candidate in autoimmune and/or inflammatory diseases. These result let us to think that the gene variation of FOXJ1 might be associated with susceptibility to allergic and/or autoimmune diseases. Through the results of our previous study, we confirmed that the polymorphisms of $g .-460 C>T, 1805 G>T, g .3375 G>C$ in human FOXJ1 may be associated with the susceptibility to allergic rhinitis in a Korean population (Li et al., 2006). Moreover, our present studies indicate that $g .3375 G>C$ could be specifically associated with the susceptibility to SLE (Table 1). Interestingly, the genome-wide screen study from three ethnic groups indicates that the human FOXJ1 gene is located on chromosome 17q22-25 region linked with SLE (Cantor et al., 2004). The polymorphism of $g .3375 \mathrm{G}>\mathrm{C}$ falls on $3^{\prime}-\mathrm{UTR}$ region that is particularly considered to be engaged in the control of translation, mRNA stability and poly-adenylation of signaling molecules (Kuersten and Goodwin, 2003). The genotype frequencies of FOXJ1 gene polymorphisms are significantly different between SLE male patients and the male control group, but no difference between both female groups. These results indicated that the male with $\mathrm{g} .3375 \mathrm{G}>\mathrm{C}$ polymorphism in FOXJ1 are more significantly associated with the susceptibility of SLE than female, and suggest that the association of the SNPs of FOXJI (g.1805G $>T$ and $g .3375 G>C$ ) could be affected by the gender of SLE patients. However, it 
will be need to validate using large male's SLE samples by other research group. It is difficult to enroll SLE male patients. Because of SLE are very rare disease and the sex ratio between female and male in SLE patients is 9:1.

A hallmark of SLE and RA is the presence of autoantibodies; therefore further evaluation was made to see these SNPs have associations with ANA, RF and anti-CCP. The association levels were measured by ANOVA and compared the relationship. The genotype of FOXJ1 gene polymorphisms have no significant association with the RF and anti-CCP levels in RA patients and also no significant association with the ANA levels in SLE patients (Table 3). We also analyzed each genotype of these SNPs with RNP levels and hemolytic anemia in SLE patients. But there are also no significant associations by each genotype (data not shown). These results suggest that the polymorphisms of the FOXJ1 are not associated with these factors in RA or SLE patients. The haplotype distribution, specifically main haplotype $C G G$, by g. $-460 C>T$, g.1805G $>T$ and $g .3375 C>T$ polymorphisms of FOXJ1 gene between the healthy controls and RA patients were significantly different, and the haplotype CTG also showed the significant difference between SLE patients and controls (Table 4). This result suggests that the haplotype by FOXJ1 gene polymorphisms might be one of the most important genetic factors in RA and SLE susceptibility.

In summary, the $9.3375 G>C$ polymorphisms of FOXJ1 might be contribute to the genetic predisposition to autoimmune diseases. These findings demonstrate that SLE and RA may have not a common genetic background in the case of human FOXJ1 gene. Because some FOXJ1 variants appears to be unique only for SLE. It is interesting to know that FOXJ1 polymorphism $(g .3375 G>C)$ might have some influence on susceptibility to autoimmune diseases. Thus, our results will be important in future studies, to determine whether or not this polymorphism affects the FOXJ1 gene translation, mRNA stability and function.

\section{Acknowledgement}

This work was supported by a grant from the Korea Health 21 R\&D Project by Ministry of Health and Welfare (A010251).

\section{References}

Abbas AK, Murphy KM, Sher A. Functional diversity of helper T lymphocytes. Nature 1996;383:787-93

Akahoshi M, Nakashima H, Tanaka Y, Kohsaka T, Nagano S, Ohgami E, Arinobu Y, Yamaoka K, Niiro H, Shinozaki M,
Hirakata H, Horiuchi T, Otsuka T, Niho Y. Th1/Th2 balance of peripheral Thelper cells in systemic lupus erythematosus. Arthritis Rheumatism 1999;42:1644-8

Arnett FC, Edworthy SM, Bloch DA, McShane DJ, Fries JF, Cooper NS, Healey LA, Kaplan SR, Liang MH, Luthra HS, et al. The American Rheumatism Association 1987 revised criteria for the classification of rheumatoid arthritis. Arthritis Rheum 1988;31:315-24

Blatt EN, Yan XH, Wuerffel MK, Hamilos DL, Brody SL. Forkhead transcription factor $\mathrm{HFH}-4$ expression is temporally related to ciliogenesis. Am J Respir Cell Mol Biol 1999; 21:168-76

Cantor RM, Yuan J, Napier S, Kono N, Grossman JM, Hahn $\mathrm{BH}$, Tsao BP. Systemic lupus erythematosus genome scan: support for linkage at $1 q 23,2 q 33,16 q 12-13$, and 17q21-23 and novel evidence at 3p24, 10q23-24, 13q32, and 18q2223. Arthritis Rheum 2004;50:3203-10

Carlsson P, Mahlapuu M. Forkhead transcription factors: key players in development and metabolism. Dev Biol 2002;250: 1-23

Chae SC, Song JH, Pounsambath P, Yuan HY, Lee JH, Kim JJ, Lee YC, Chung HT. Molecular variations in Th1-specific cell surface gene Tim-3. Exp Mol Med 2004;30:274-8

Chang DM, Su WL, Chu SJ. The expression and significance of intracellular T helper cytokines in systemic lupus erythematosus. Immunol Invest 2002;31:1-12

Chen J, Knowles HJ, Hebert JL, Hackett BP. Mutation of the mouse hepatocyte nuclear factor/forkhead homologue 4 gene results in an absence of cilia and random left-right asymmetry. J Clin Invest 1998;102:1077-82

Coffer PJ, Burgering BMT. Forkhead-box transcription factors and their role in the immune system. Nat Rev Immunol 2004;4:889-99

Dolhain RJ, van der Heiden AN, ter Haar NT, Breedveld FC, Miltenburg AM. Shift toward T lymphocytes with a T helper 1 cytokine-secretion profile in the joints of patients with rheumatoid arthritis. Arthritis Rheum 1996;39:1961-9

Gregersen PK. Genetics of rheumatoid arthritis: confronting complexity. Arthritis Res 1999;1:37-44

Hackett BP, Brody SL, Liang M, Zeitz ID, Bruns LA, Gitlin JD. Primary structure of hepatocyte nuclear factor/forkhead homologue 4 and characterization of gene expression in the developing respiratory and reproductive epithelium, Proc Natl Acad Sci USA 1995;92:4249-53

Harrison B, Thomson W, Symmons D, Ollier B, Wiles N, Payton T, Barrett E, Silman A. The influence of HLA-DRB1 alleles and rheumatoid factor on disease outcome in an inception cohort of patients with early inflammatory arthritis. Arthritis Rheum 1999;42:2174-83

Ho IC, Glimcher LH. Transcription: tantalizing times for T cells. Cell 2002;109:S109-20

Hochberg MC. Updating the American College of Rheumatology revised criteria for the classification of systemic lupus erythematosus. Arthritis Rheum 1997;40:1725

Hochberg MC. The epidemiology of systemic lupus erythe- 
matosus. In: Dubois' lupus erythematosus (Wallace DJ, Hahn BH eds), 1997, 49-65, Williams and Wilkins, Baltimore

Kaestner KH, Knochel W, Martinez DE. Unified nomenclature for the winged helix/forkhead transcription factors. Genes Dev 2000;14:142-6

Kotzin B. Systemic lupus erythematosus. Cell 1996;85:303-

Kuchroo VK, Das MP, Brown JA, Ranger AM, Zamvil SS, Sobel RA, Weiner HL, Navabi N, Glimcher LH. B7-1 and B7-2 costimulatory molecules differentially activate the $\mathrm{TH} 1 / \mathrm{TH} 2$ developmental pathways: application to autoimmune disease therapy. Cell 1995;80:707-16

Kuersten S, Goodwin EB. The power of the 3' UTR: translational control and development. Nat Rev Genet 2003;4: 626-37

Lafaille JJ, Keere FV, Hsu AL, Baron JL, Haas W, Raine CS, Toneqawa S. Myelin basic protein-specific T helper 2 (Th2) cells cause experimental autoimmune encephalomyelitis in immuno-deficient hosts rather than protect them from the disease. J Exp Med 1997;186:307-12

Li CH, Chae SC, Lee JH, Zhang QG, Chung HT. Identification of single nucleotide polymorphisms in FOXJ1 and their association with allergic rhinitis. J Hum Genet 2006; 51:292-7

Lim L, Zhou H, Costa RH. The winged helix transcription factor HFH-4 is expressed during choroid plexus epithelial development in the mouse embryo. Proc Natl Acad Sci

\section{USA 1997; $94: 3094-9$}

Lin L, Spoor MS, Gerth AJ, Brody SL, Peng SL. Modulation of Th1 activation and inflammation by the NF-kappaB repressor Foxj1. Science 2004;303:1017-20

Lin L, Brody SL, Peng SL. Restraint of B cell activation by Foxj1-mediated antagonism of NF-kappaB and IL-6. J Immunol 2005;175:951-8

Mosmann TR, Coffman RL. TH1 and TH2 cells: different patterns of lymphokine secretion lead to different functional properties. Annu Rev Immunol 1989;7:145-73

Schellekens GA, Visser $\mathrm{H}$, de Jong BA, van den Hoogen $\mathrm{FH}$, Hazes JM, Breedveld FC, van Venrooij WJ. The diagnostic properties of rheumatoid arthritis antibodies recognizing a cyclic citrullinated peptide. Arthritis Rheum 2000;43:155-63

Simon AK, Seipelt E, Sieper J. Divergent T-cell cytokine patterns in inflammatory arthritis. Proc Natl Acad Sci USA 1994;91:8562-6

Tran H, Brunet A, Griffith EC, Greenberg ME. The many forks in FOXO's road. Sci STKE 2003;272:RE5

Van der Heide A, Jacobs JW, Haanen HC, Bijlsma JW. Is it possible to predict the first year extent of pain and disability for patients with rheumatoid arthritis? J Rheumatol 1995; 22:1466-70

Weigel $D$, Jackle $H$. The fork head domain: a novel DNA binding motif of eukaryotic transcription factors. Cell 1990; 63:455-6 